DOI: 10.2478/ausae-2019-0001

\title{
First molecular identification of wheat seed gall nematode Anguina tritici races parasitized on wheat in Iraq
}

\author{
Sulaiman Naif AMI, ${ }^{1}$ Ibrahim Esa TAHER, ${ }^{1}$ \\ Fenik Sherzad HUSSEN, ${ }^{2}$ Ayoub Ibrahim AHMED ${ }^{3}$ \\ ${ }^{1}$ Department of Plant Protection, College of Agricultural Engineering Sciences, \\ University of Duhok, Kurdistan Region - Iraq, e-mail: sulaiman.ami@uod.ac \\ *Corresponding author: Ibrahim.esa@uod.ac \\ ${ }^{2}$ Department of Biology, College of Science, University of Salahaddin, \\ Kurdistan Region - Iraq, e-mail: fenik_aya@yahoo.com \\ ${ }^{3}$ Department of Plant Protection, Technical Institute in Khabat, Erbil Polytechnic University, \\ Kurdistan Region - Iraq, e-mail: ayoub.ibrahim@epu.edu.krd
}

Manuscript received December 07, 2018; revised January 23, 2019; Accepted January 30, 2019

\begin{abstract}
This study was conducted to identify races of wheat seed gall nematode Anguina tritici by using molecular identification for this nematode species parasitized on durum and bread wheat cultivars. Wheat seed galls of both cultivars were collected from two cities, Erbil and Duhok, from the Kurdistan Region and Iraq respectively. DNA was extracted from both nematode isolates (populations), and then PCR reactions were performed with Internal Transcribed Spacer (ITS) region using primers TW81/AB28 with $2 \mu$ l of template DNA of $A$. tritici for both nematode isolates on both wheat cultivars. The bands of both amplification products of PCR reactions were visualized in position about $800 \mathrm{bp}$ on agarose gel, which indicates that both isolates of A. tritici belong to the same race. On the other hand, no nucleotide differences were observed between the two nematode isolations, as revealed by the sequence alignment of DNA of the internal transcribed spacer (ITS) region and phylogenetic tree, which emphasizes at the same time that both nematode isolates are of the same genetic structure or have the same identity and confirms their belonging to the same nematode race.
\end{abstract}

Keywords: molecular identification, Anguina tritici, races, wheat 


\section{Introduction}

Ear-cockle disease is the oldest disease of wheat (Triticum spp.) [1], which was caused by the first recorded plant parasitic nematode in England by John Needham in 1743 - that is, wheat seed gall nematode Anguina tritici (Steinbuch, 1799; Filipjev, 1936) [2]. It is one of the major aerial diseases, and it causes sustainable losses in the wheat crop of tropical and sub-tropical countries [3]. It can be found in any place where wheat is grown, and this disease is still common in Eastern Europe and in parts of Africa and Asia [4]. Symptoms of nematode attack can be discerned at the seedling stage, but farmers generally fail to recognize the disease before harvesting and threshing of the plant [5]. Since its first record in Iraq by Rao in 1921 [6], A. tritici has remained an important nematode pest in Iraq, occurring in most wheat-growing areas, with a disease incidence from $22.9 \%$ up to $45 \%$ on the mexipac cv. of wheat [4]. A. tritici caused reduction in the wheat yield, reaching $57 \%$ in mexipak cv. [7], which increased to $75 \%$ on the same cultivar in Duhok Province in 1989 [8]. Ami et al. [9] reported that the percentage of infestation by galls reached its maximum value $(50 \%)$ in bread wheat in Bashika, northern Iraq. Ear-cockle disease reduces the human consumption and market price of wheat [10], with significant reduction in the protein and gluten contents of the flour product of infested wheat with seed galls [11]. During the survey in Duhok Province in 2010-2011, it was shown that A. tritici was still one of the major problems in this region, reaching 50\% in some wheat fields [12].

It is obvious from the previous studies that there are three races of A. tritici isolated from wheat plants that could not infect barley plants $[11,12,13]$. So, it was considered that there is another race on barely. Stephan et al. [14] stated that galls from durum wheat cultivars attacked only durum cultivars and from bread wheat infected only bread wheat to show the existence of durum wheat race and bread wheat race. In addition, another race of $A$. tritici was collected in 1998 and recorded for the first time by Stephan et al. [15] based on the differences of their obvious effects on wheat plants such as spike number and seed production. On the other hand, Taher [12] found that the A. tritici population from bread wheat has the ability to infect many durum cultivars. Therefore, this study aimed to clarify if there is any variation or similarity in the genetic structure of both populations of wheat seed gall nematode A. tritici parasitized on durum and bread wheat to verify - by using molecular identification technique - whether they belong to the same race or to two different races. 


\section{Materials and methods}

\section{A. Nematode samples}

Two isolates (populations) of wheat galls were collected, involving galls of durum wheat from the Erbil silo in Erbil Province and galls of bread wheat from the Faydiyi silo in Duhok Province. These two different isolates were used as a source of A. tritici for nematode DNA extraction.

\section{B. The extraction and purification of deoxyribonucleic acid (DNA)}

DNA were extracted from several $2^{\text {nd }}$-stage juveniles (Fig. 1) for each nematode isolate from Erbil and Duhok provinces, using worm Lysis buffer (WLB), which consisted of $10 \mathrm{mM}$ Tris $\mathrm{pH} 8.2,50 \mathrm{mM} \mathrm{KCl}, 0.45 \%$ Tween 20, $2.5 \mathrm{mM} \mathrm{MgCl}$, $0.05 \%$ gelatine, and $60 \mu \mathrm{g} / \mathrm{ml}$ Proteinase $\mathrm{K}$. Nematode juveniles were crushed on the clean slide with $10 \mu \mathrm{l}$ WLB under binocular microscopes and then transferred to a new PCR tube on ice with an extra $10 \mu \mathrm{l}$ of WLB. The samples were frozen at $-80^{\circ} \mathrm{C}$ for 10 minutes, and then samples were warmed up to room temperature, after which incubated in water bath at $60{ }^{\circ} \mathrm{C}$ for $1 \mathrm{~h}$ and followed by a $95{ }^{\circ} \mathrm{C}$ incubation for $10 \mathrm{~min}$ to completely lyse the cells, digest the proteins, and inactivate proteinase K. Subsequently, the tube was cooled on ice and centrifuged at $6,000 \mathrm{rpm}$ for $30 \mathrm{sec}$. [16]. The supernatant material containing the DNA was gathered and stored at $-20^{\circ} \mathrm{C}$ or directly used for PCR.

\section{The amplification of target nucleic acid DNA}

Two primers were used for the amplification of the ITS-rRNA gene, which were TW81 (5'-GTTTCCGTAGGTGAACCTGC-3') and AB28 (5'ATATGCTTAAGTTCAGCGGGT-3') for both nematode populations. The amplification was performed in $25 \mu \mathrm{l}$ reactions containing $12.5 \mu \mathrm{l}$ Red MyTaq $^{\mathrm{TM}}$ (Mix Master Mix), $1 \mu 1$ of each primer, and the $2 \mu 1$ of DNA template with $8.5 \mu \mathrm{l}$ of a double distilled water to obtain a final volume of $25 \mu \mathrm{l}$. The conditions of the PCR reaction were $95^{\circ} \mathrm{C}$ for $4 \mathrm{~min}$, followed by 35 cycles of $95^{\circ} \mathrm{C}$ for $40 \mathrm{sec}, 56.5^{\circ} \mathrm{C}$ for $40 \mathrm{sec}, 72^{\circ} \mathrm{C}$ for $1 \mathrm{~min}$, and a final extension of 72 ${ }^{\circ} \mathrm{C}$ for $10 \mathrm{~min}$ [17]. The amplified products and a phiX174 DNA / HaeIII marker were separated on a $1 \%$ agarose gel stained with Gel Red in $1 \times$ TAE and then examined under UV light.

\section{DNA sequencing}

This process was performed in the laboratories of My TACG Bioscience Genomics BioSci and Tech Company in Malaysia, where samples of amplified 
DNA products for both nematode isolations were sent to determine the order of the four nitrogenous bases, including: Adenine (A), Guanine (G), Cytosine (C), and Thymine $(\mathrm{T})$ in a strand of DNA. The BioEdit program was used for alignment and a sequence scanner to check the sequence quality before the alignment (http://www.mbio.ncsu.edu/bioedit/bioedit.html).

After the submission sequences of $A$. tritici for both nematode isolates to GenBank in NCBI, the accession number was determined, which was for the Erbil isolate KT900694 while for the Duhok isolate KT900693.
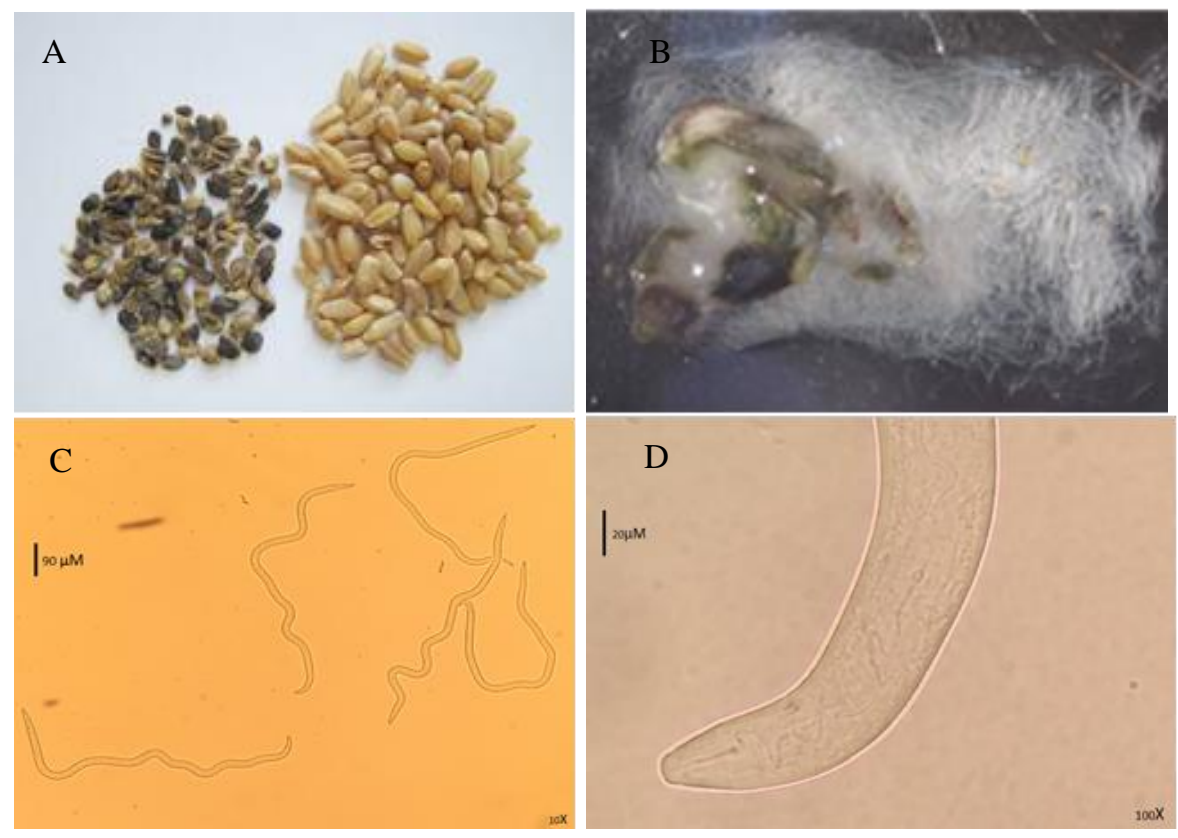

Figure 1. Healthy wheat seeds and galls (A), exiting of second-stage juveniles

(J2) of A. tritici after opening the gall (B), free $\mathrm{J} 2$ of $A$. tritici $(\mathrm{C})$, and anterior portion of $\mathrm{J} 2$ show stylet and esophagus (D)

\section{E. Extraction of phylogenic tree}

Phylogenic tree was extracted applying the NCBI BLAST program (https://www.ncbi.nlm.nih.gov/BLAST/) by which all nematode species that have the required percentage of genetic similarity with the studied nematode $A$. tritici can be obtained, and it is worth mentioning that those nematodes are in themselves species of the same genus or the same family, and by choosing some of them phylogenic tree was extracted. 


\section{Results and discussions}

DNA sequencing of the Internal Transcribed Spacer ITS PCR-amplified product revealed a size of $800 \mathrm{bp}$ among both isolates of wheat seed gall nematodes $A$. tritici from Erbil and Duhok provinces.

Fig. 2 illustrates the typical amplification products of PCR reactions with primers TW81/AB28, using $2 \mu 1$ of template DNA of $A$. tritici for both isolates of Erbil and Duhok provinces. The bands of both amplification products of PCR reactions were visualized in position at about 800 bp on agarose gel, which indicates that both isolates of $A$. tritici from Erbil and Duhok provinces belong to the same race because they have the same size of bp. and genotype.

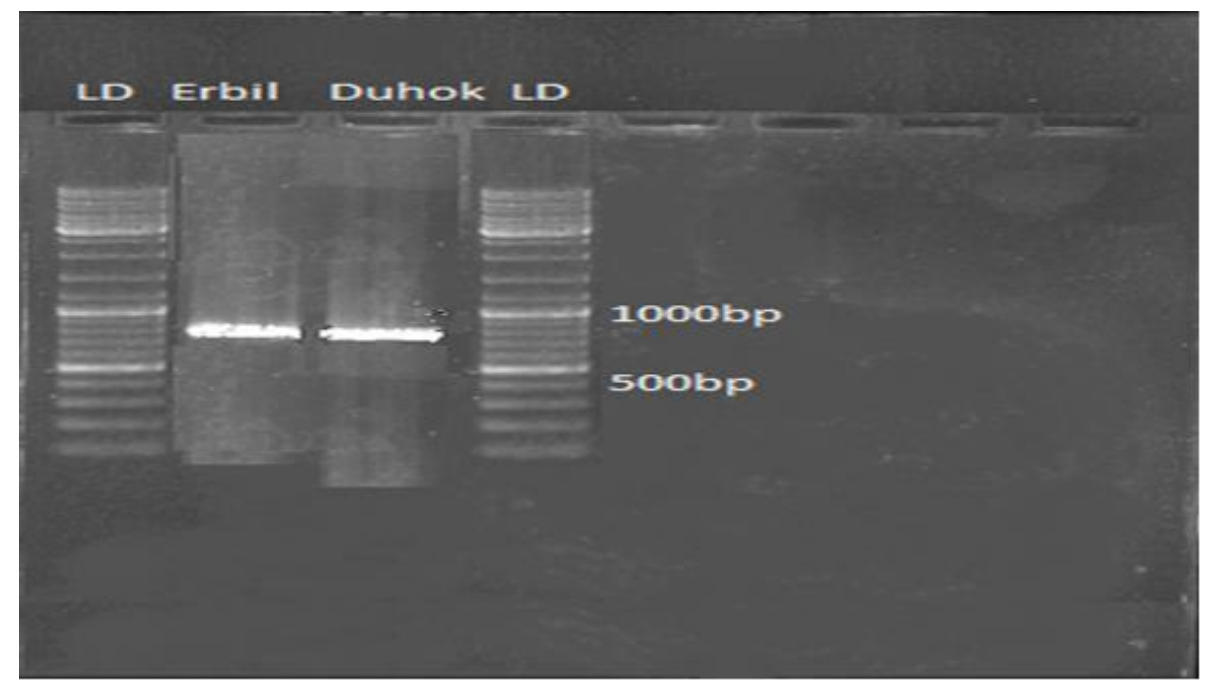

Figure 2. Agarose gel shows typical amplification products of PCR reactions with primers TW81/AB28, using $5 \mu$ l of template DNA of Anguina tritici of both isolates $\mathrm{AE}$ and $\mathrm{AD}$ from Erbil and Duhok provinces respectively.

On the other hand, no nucleotide differences were observed between either of the isolations by comparing the DNA sequence alignment of the internal transcribed spacer (ITS) region for both nematode isolates, as clarified in Fig. 3 and Fig. 4. Their genetic similarity analysis, as illustrated by the phylogenetic tree (Fig. 5), revealed that both nematode isolates are of the same genetic structure or have the same identity, which also confirms their belonging to the same nematode race. 


$\begin{array}{lllcl}\text { Score } & \text { Expect } & \text { Identities } & \text { Gaps } & \text { Strand } \\ 1338 \text { bits }(724) & 0.0 & 724 / 724(10096) & 0 / 724(096) & \text { Plus } / \text { Plus }\end{array}$

Query 1 ACCTGCTCCCGGATCATTACCGATCAACCTGAAAACTAGCGGGAGGACCTGCCTGGACCT 60 Sbjet 1 ACCTCCTGCCGGATCATTACCGATCAACCTGAAAACTACCCGGAGGACCTCCCTGCACCT 60 Query 61 CCTCTGTAGAATGACGACTTCTTCATTCCTACACCCAATACCTCAAGAGCGICCCGTGAT 120 Sbjat 61 CCTCTGTAGAATGACGACTTCTTCATTCCTACAGCCAATAGCTCAAGAGCGTGCCGTGAT 120 Query 121 ATTGCCATGCTGCTIACAGGTGACGTCCCCACCGACTACCAGCCTTATTCTTGGGCGAAA 180 Sbjet 121 ATTGCCATGCTGCTTACAGGTGACGTCCCCACCGACTAGCAGCCTTATTCTTGGCCGAAA 180 Query 181 AACGCCTTAGTTGCTTCTAAGTTCTCTGAGCAGTTGTATCCCTACGICCGTGCCTCCG 240 Sbjet 181 AACGCCTTAGTTGCCTTCTAAGTTTCTCTGAGCAGTTTATCCCTACGTCCGTGCCTCCG 240 Qvery 241 TTGAAGAGAAACGGTACGTGGTCTTCGTGATCGCGAGAATTAATGACCCCCAGATGTGGT 300 Sbjet 241 TTGAAGAGAAACCGTACGTCGTCTTCGTGATCCCGAGAATTAATGAGCGCCAGATGTGO 300 Query 301 GCCCCCAACAAAACAACCATTTTGAACTTTTGAGAAATAACATTTCTAGTCTTACCGG 360 Sbjet 301 GCCCCCAACAAAACAACCATTTTGAACTTTTTGAGAAATAACATTTCTAGTCTTACCCG 360 Query 361 TGGATCACTCGOTTCATAGATCGATGAAGAACGCAGCCAACTGCGATATATGGTGTGAAC 420 Sbjet 361 TGGATCACTCGGTTCATAGATCGATGAAGAACCCACCCAACTGCGATATATGGTGTGAAC 420 Query 421 TCCAGATATTTGAACACCAAGAATTCGAATCCACATTCCGCCACTGGATATTTATCCTT 480 Sbjet 421 TCCAGATATTTGGACACCAAGAATTCGAATCCACATTGCCCCACTGGATATTTATCCTT 480 Query 481 TGCCACATCTCCCTCAGGGTCGTAAACACTAAACGAAACCTATTCGTTGTTATGACAG4 540 Sbjet 481 TCGCACATCTCCCTCAGCGTCGTAAACACTAAACGAAAGCTATTCGTTGTTATGACAGA 540 Qvery 541 CTCATGGCTACACTAGTTAGGGCGATATTCCGCTAGAGTCATGTTTCTGTGAAGTGCITT 600 Sbjet 541 CTCATGCCTACACTAGTTAGGGGGATATTCCCCTAGAGTCATGTTTCTGTGAAGTGGTT 600 Query 601 TGCCTACCGGTTCCCTACGCCCGTCTCATCATCATGTCTTCCCTAGTGTAGACGTATCTG 660 Sbjet 601 TCCCTACCCGTTCCCTACGCCCGTCTCATCATCATGTCTTCCCTAGTGTAGACGTATCTG 660 Quey 661 ATGGCTGTACCACATCGATTACATGTAGCCATGGATCTTCCGACCTGAGCTCAGGTGTGA 720 Sbjet 661 ATGGCTGTACCACATCGATTACATGTAGCCATGGATCTTCCGACCTGAGCTCAGGTGTGA 720 Query 721 TCAC 724

Sbjet 721 TCAC 724

Figure 3. Anguina tritici isolate AE Internal Transcribed Spacer1, Partial Sequence; 5.8S Ribosomal RNA gene, Complete Sequence; and Internal Transcribed Spacer 2, partial sequence. Sequence ID: KT900694.1; Length: 724; Number of Matches: 1 


\begin{tabular}{|c|c|c|c|c|}
\hline Score & Expect & Identities & Gaps & Strand \\
\hline 1338 bits(724) & 0.0 & $724 / 724(100 \%)$ & $0,724(0 \% 6)$ & Plus / Plus \\
\hline
\end{tabular}

Query 1 ACCTGCTGCCGGATCATTACCGATCAACCTGAAAACTAGGGGGAGGACCTGCCTGGACCT 60

Sbjet 1 ACCTGCTGCCGGATCATTACCGATCAACCTGAAAACTAGGGGGAGGACCTGCCTGGACCT 60

Query 61 CCTCTGTAGAATGACGACTTCTTCATTCCTACAGCCAATAGCTCAAGAGGGTGCCGTGAT 120

Sbjet 61 CCTCTGTAGAATGACGACTTCTTCATTCCTACAGCCAATAGCTCAAGAGGGTGCCGTGAT 120

Query 121 ATTCCCATCCTCCTTACAGGTGACGTCCCCACCGACTAGCAGGCTTATTCTTGGGCGAAA 180

Sbjet 121 ATTGGCATGCTGCTTACAGGTGACGTCCCCACCGACTACCAGCCTTATTCTTGGGCGAAA 180

Query 181 AACGCCTTAGTTGCTTCTAAGTTCTCTGACCAGTTGTATGCCTACGTCCGTGGCTGCG 240

Sbje 181 AACGCCTTAGTTCCCTTCTAAGTTCTCTGAGCAGTTGTATCCCTACGTCCGTGCCTCCG 240

Qury 241 TTGAAGAGAAACGGTACGTGGTCTTCGTGATCGCGAGAATTAATGAGCGCCAGATGTGGT 300

Sbja 241 TTGAAGAGAAACGTACGTGGTCTTCGTGATCGCGAGAATTAATGAGCCCCAGATGTGGT 300

Qvery 301 GCCGCCAACAAAACAACCATTTTTGAACTTTTTGAGAAATAACATTTCTAGTCTTACCCG 360

Sbja 301 GCCCCCAACAAAACAACCATTTTGAACTTTTGAGAAATAACATTTCTAGTCTTACCGG 360

Qvery 361 TGGATCACTCGGTTCATAGATCGATGAAGAACCCAGCCAACTGCGATATATGGTGTGAAC 420

Sbje 361 TGGATCACTCGGTTCATAGATCGATGAAGAACGCAGCCAACTCCGATATATGGTGTGAAC $\quad 420$

Qvery 421 TGCAGATATTTGAACACCAAGAATTCGAATCCACATTGCGCCACTGGATATTTATCCTT 480

Sbje 421 TCCAGATATTTGAACACCAAGAATTCGAATGCACATTGCGCCACTGGATATTTATCCTT 480

Query 481 TCCCACATCTCCCTCAGGGTCGTAAACACTAAACGAAAGCTATTCGTTGTTATGACAGA 540

Sbja 481 TGGCACATCTGGCTCAGGGTCGTAAACACTAAACGAAAGCTATTCGTTGTTTATGACAGA 540

Query 541 CTCATGCCTACACTAGTTAGGGGGATATTCCCCTAGAGTCATGTTTCTGTGAAGTCGTIT 600

Sbja 541 CTCATCCCTACACTAGTAGGGGGATATTCCGCTAGAGTCATGTTTCTGTGAAGTGGTT 600

Query 601 TGCCTACCGTTGCCTACGGCCGTCTCATCATCATGTCTGGCTAGTGTAGACGTATCTG 660

Sbja 601 TGCCTACCGGTGCCTACGGCCGTCTCATCATCATGTCTTGGCTAGTGTAGACGTATCTG 660

Query 661 ATGGCTGTACCACATCGATTACATGTAGCCATGGATCTTCCGACCTGACCTCAGGTGTGA 720

Sbje 661 ATGGCTGTACCACATCGATTACATGTAGFCATGGATCTTCCGACCTGAGCTCAGGTGTGA 720

Query 721 TCAC 724

Sbjet 721 TCAC 724

Figure 4. Anguina tritici isolate AD Internal Transcribed Spacer1, Partial Sequence; 5.8S Ribosomal RNA gene, Complete Sequence; and Internal Transcribed Spacer 2, Partial Sequence. Sequence ID: KT900693.1; Length: 724; Number of Matches: 1

Phylogenetic tree (Fig. 5) was extracted to determine the amount of genetic change per time unit between both nematode isolates and some other isolates and species of Anguina as well as some nematode species belonging to the family Anguinidae such as stem and bulb nematodes Ditylenchus since they belong to the same ancestors. 


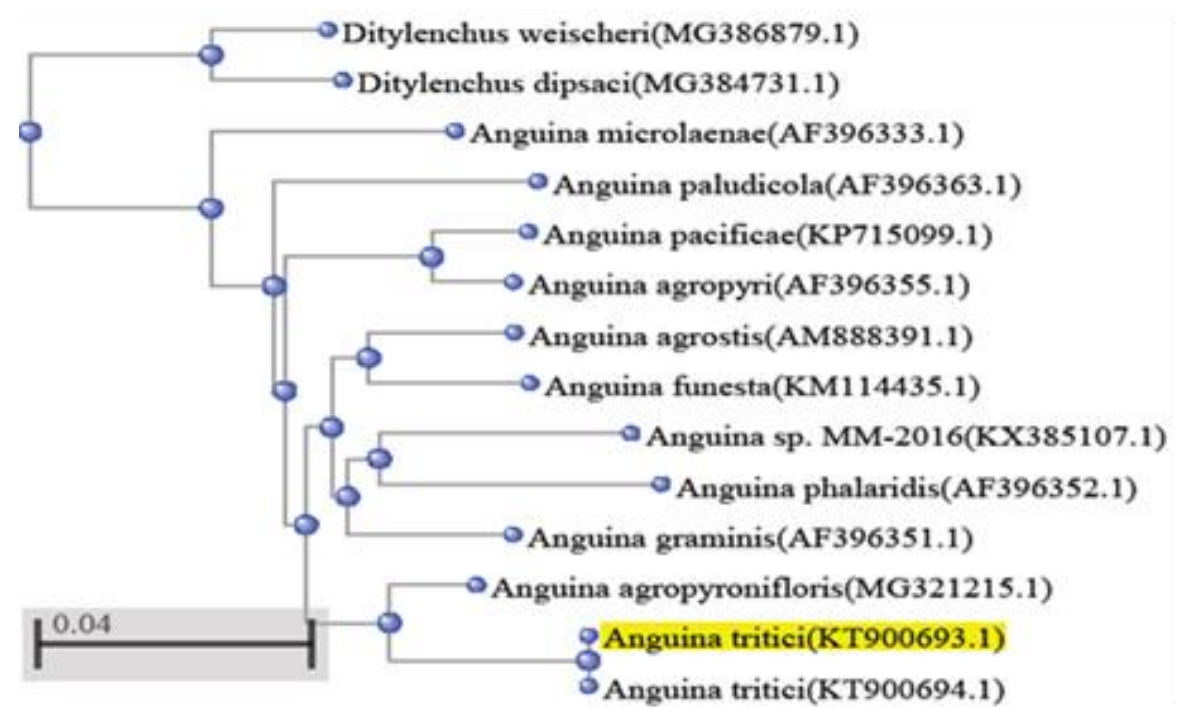

Figure 5. Best Maximum Likelihood Phylogenetic tree based on rDNA ITS sequences within isolates and some species of Anguina and two species of Ditylenchus. Branch lengths are proportional to the number of inferred changes.

The horizontal lines or branches in this tree represent an evolutionary pedigree that changes over time: the longest the branch in the horizontal dimension, the largest the amount of alteration. The scale bar at the base of the figure displays a scale for this. In this case, the line segment with the number 0.04 shows the length of branch that represents an amount of genetic alteration by 0.04 . The units of branch length are nucleotide substitutions per site, which is the number of changes or substitutions divided by the sequence length. The vertical lines are used to lay out the tree visual, and therefore they simply show the connection of horizontal lines with each other and for how long they are unpertinent.

It seems from the phylogenetic tree that the closest species to both nematode isolates is A. agropyroniflori with a genetic similarity of $95 \%$, while nucleotide differences increased with other nematode species where more nucleotide differences were observed with $D$. dipsaci, in which the genetic similarity between both isolates and this species reached $86 \%$ (Table 1). 
Table 1. Genetic similarity percentage of isolates $\mathrm{AE}$ and $\mathrm{AD}$ of wheat seed gall nematode A. tritici, which have the accession numbers of KT900694 and KT900693, respectively, with some other nematode species composition [\%] of the selected essential oils [13]

\begin{tabular}{lcc}
\hline \multicolumn{1}{c}{ Nematode species } & $\begin{array}{c}\text { Accession number } \\
\text { or sequence ID }\end{array}$ & $\begin{array}{c}\text { Genetic similarity } \\
\text { percentage with both } \\
\text { isolates of } \text { A. tritici }\end{array}$ \\
\hline A. agropyronifloris & MG321215 & 95 \\
A. agrostis & AM888391 & 92 \\
A. pacificae & KP715099 & 91 \\
A. funesta & KM114435 & 91 \\
A. obesa & KX385107 & 91 \\
A. graminis & AF396351 & 92 \\
A. agropyri & $\mathrm{AF} 396355$ & 91 \\
A. phalaridis & $\mathrm{AF} 396352$ & 90 \\
A. paludicola & $\mathrm{AF} 396363$ & 90 \\
A. microlaenae & $\mathrm{AF} 396333$ & 90 \\
Ditylenchus weischeri & $\mathrm{MG} 386879$ & 87 \\
D. dipsaci & $\mathrm{MG} 384731$ & 86 \\
\hline
\end{tabular}

This results clarified that all research related to wheat seed gall nematode $A$. tritici on wheat in Iraq which considered that both durum wheat isolate and bread wheat isolate of that nematode to be different races are not really accurate. Both isolates are the same, but the infection of durum or bread wheat by this nematode is due to the susceptibility of wheat cultivars, environmental circumstances, planting date, the application of both pesticides and herbicides by farmers, and the agricultural processes that one way or another affect the pathogenicity of $A$. tritici $[11,12]$.

Taher [12] rejected the idea that nematodes isolated from bread wheat invaded only bread wheat cultivars and those from durum wheat attacked only durum wheat cultivars. Thus, several races had been recorded by Stephan et al. [14] in addition to another new race of $A$. tritici, collected in 1998 and recorded for the first time by Stephan et al. [15] based on differences in their pathogenicity on wheat plants such as spike number and seed production. 
These three races mentioned by previous studies do not resemble the $\mathrm{J}_{2}$ population - used in the experiment carried out by Taher [12] - regarding its behaviour of infection, and this might indicate new races of $A$. tritici which have ability to infect different cultivars of durum and bread wheat but incapable of infecting barley cultivars. This result was obtained by Mustafa [11], who reported that $A$. tritici isolated from wheat plants could not parasite on barley plants. Al-Talib et al. [13] recorded a new race of barely plant named barley race. It is clear that there are two races of wheat and barley, but this molecular study emphasized for the first time that both nematode isolates from durum and bread wheat have the same genetic characteristics depending on the amplification products of PCR reactions. Therefore, we can say that A. tritici consists of two races, including wheat and barley races, while the pathogenicity of wheat race varies by different cultivars of wheat as well as the infection behaviour and severity may change in different regions and under different environmental circumstances. Also, we recommend the implementation of further molecular studies on different populations for the further clarification of these nematode races in Iraq. According to this result, A. tritici on durum and bread wheat belong to the same race or one race, which means that there are two races of $A$. tritici in Iraq: one on wheat and the other one on barley.

\section{Conclusions}

Molecular identification of wheat seed gall nematode Anguina tritici parasitized on durum and bread wheat cultivars which were collected from two cities, Erbil and Duhok, from the Kurdistan Region and Iraq, respectively, emphasized that both nematode isolates are of the same genetic structure or have the same identity and confirmed their belonging to the same nematode race.

\section{References}

[1] Bhatti, S., Dahiya, R. S., Dhawan, S. C. (1978), New record of tundu and ear-cockle incidence in barley. Nematol., 331-332.

[2] Perry, R. N., Moens, M. (2006), Plant nematology. British Library, London, UK: CAB International.

[3] Kort, J. (1972), Nematode diseases of cereals of temperate climates. In: J. M. Webster (ed.), Economic Nematology, 97-126.

[4] Al-Beldawi, A. S., Stephan, Z. A., Alwan, H. (1974), General survey of wheat gall disease in Iraq. Iraq Agric. Bull. 29, 48-69. 
[5] Khan, M. R., Athar, M. (1996), Response of wheat cultivars to different inoculum levels of Anguina tritici. Nematol. Medit. 24, 269-272.

[6] Rao, R. S. R. (1921), A preliminary list of insect pest of Iraq. Dept. of Agric. Iraq, Bull. 7.

[7] Fattah, F. A. (1988), Effect of methods on incidence of ear-cockle and tundu on wheat under field conditions. Plant and soil 109, 195-198.

[8] Stephan, Z. A., Antoon, B. G. (1990), Biotypes of earcockle nematode Anguina tritici in Iraq. Current Nematology 1, 85-8.

[9] Ami, S. N., Mohammad, H. Y., Younis, S. A. (2004), Study on ear-cockle disease in some regions of Nineveh province - North of Iraq. Iraqi J. for Agric. Sci. 5, 95-100.

[10] Paruthi, I. J., Bhatti, D. S. (1988), Effect on consumption and marketing of wheat contaminated with seed gall of Anguina tritici in Haryana. Agric. Uni. J. of Res. 18, $173-176$.

[11] Mustafa, S. A. (2009), Study on wheat and barley ear-cockle disease caused by nematode Anguina tritici in Erbil province. M.Sc. thesis, College of Agriculture, University of Salahaddin-Erbil.

[12] Taher, I. E. (2012), Pathogenicity, biology and control of wheat seed gall nematode Anguina tritici. M.Sc. thesis, College of Agriculture, University of Duhok, Duhok, Kurdistan Region, Iraq.

[13] Al-Talib, N. Y., Al-Taae, A. K. M., Nimer, S. M., Stephan, Z. A., Al-Beldawi, A. S. (1986), New record of Anguina tritici on barley from Iraq. Int. Nemat. Net. Newsl. 3, 25-27.

[14] Stephan, Z. A., Hammadi, A. I., Antoon, B. G. (1991), Races of ear-cockle nematode Anguina tritici in Iraq. Dept., State Board of Agric. Res. and Educ., Abu-Ghraib, Baghdad, Iraq. Basrah J. of Agric. Sci. 4, 1-2.

[15] Stephan, Z. A., Hassan, M. S., Hammadi, A. I., Antoon, B. G. (2000), New race of wheat gall nematode Anguina tritici and susceptibility of some cultivars to this race. Iraqi J. of Agric. 5, 1-5.

[16] Waeyenberge, L., Ryss, A., Moens, M., Pinochet, J., Vrain, T. C. (2000), Molecular characterization of 18 Pratylenchus species using rDNA restriction fragment length polymorphism. Nematol. 2, 135-142.

[17] Michel, L., Sikora, R. A. (2005), Plant parasitic nematodes in subtropical and tropical agriculture. $2^{\text {nd }}$ Edition. CABI Bioscience, Egham, UK. 